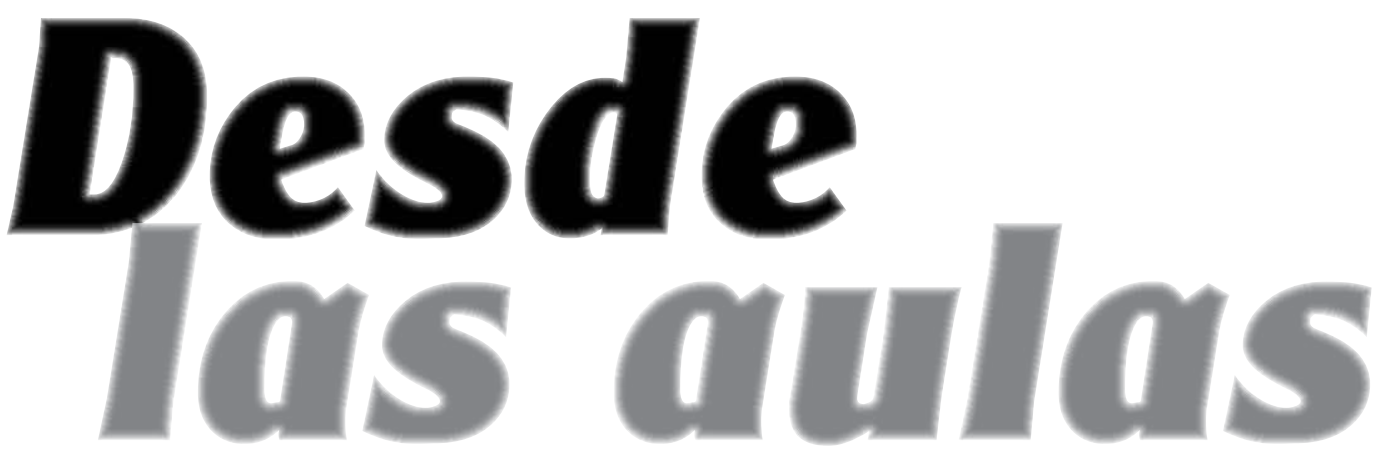

\begin{tabular}{l|l} 
& \\
& Espacio dirigido a los \\
alumnos de las diferentes \\
carreras biomédicas, tanto de \\
pregrado como de postgrado, \\
quienes deberán acompañar su \\
trabajo con lasupervisión y/o \\
asesoria de cualquiermédico \\
acreditado. De preferencia se \\
aceptarán revisiones \\
bibliográficaso proyectos \\
déinvestigación de incidencia \\
local.
\end{tabular}




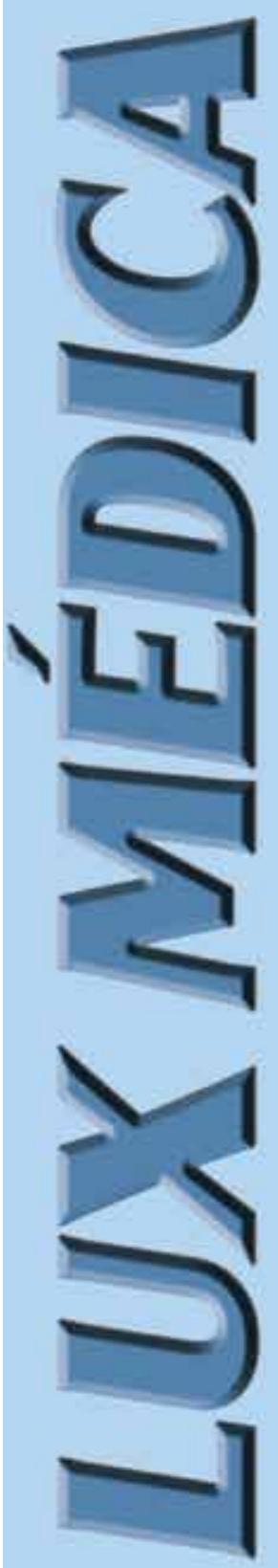




\section{Postes de fibra de vidrio como nueva opción de tratamiento en rehabilitación bucal}

Martín del Campo-Téllez Bryan Ivan*, Díaz-Alfaro Lizbeth**, Ortiz-Briones Javier Alejandro*, Komabayashi-Takashi***, Masuoka-David**.

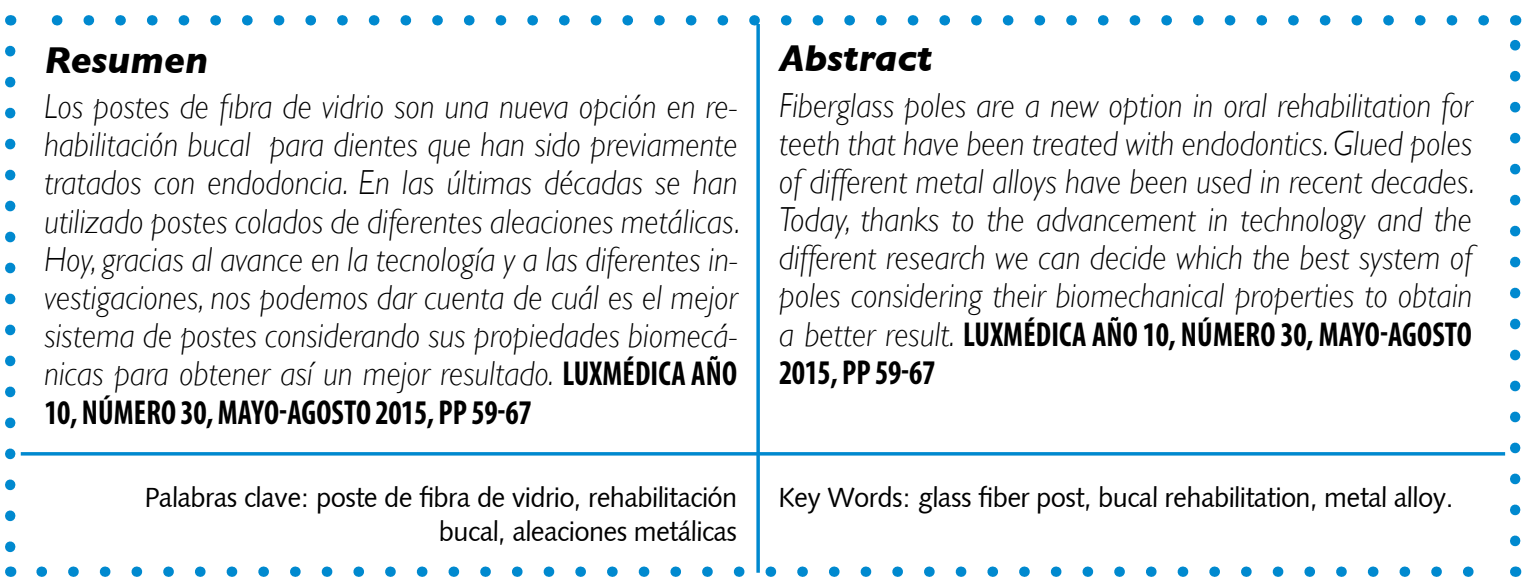

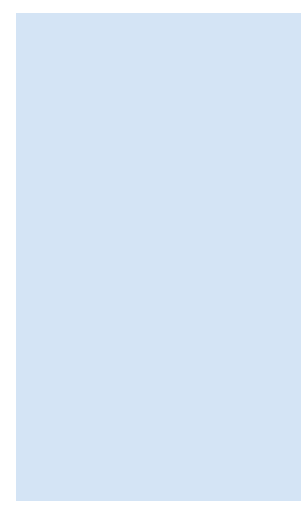

\section{Introducción}

La ciencia de los materiales dentales avanza día con día, es por eso que nos encontramos frente a nuevas alternativas para el tratamiento de un órgano dentario al que se le ha practicado una endodoncia y deseamos rehabilitarlo con una prótesis fija. La endodoncia consiste en la eliminación del paquete neurovascular o pulpa dental, y el rellenado de los espacios con materiales convencionales como la gutapercha. De acuerdo con Gutmann, cuando los cinco aspectos biológicos de la dentina se integran, una explicación razonable para que los cambios en la supuesta fortaleza de la estructura dental en los dientes sin pulpa se pueden formular. Hay

* $\quad$ Profesores investigadores del Centro de Ciencias de la Salud de la Universidad Autónoma de Aguascalientes

** Profesor investigador de la West Virginia University.

Fecha de recibido: 15 de marzo 2015

Fecha de aceptación: 7 de julio 2015

Correspondencia: Dr. David Masuoka, DDS, PhD Profesor investigador titular. Universidad Autónoma de Aguascalientes. Departamento de Estomatología. Centro de Ciencias de la Salud. Avenida Aguascalientes, No. 940. Unidad Médico Didáctica Edificio 101, Ciudad Universitaria Aguascalientes, Ags, México Código postal 20131. Teléfono +52(449)9108438 Correo electrónico david.masuoka@gmail.com 
cambios fundamentales e irreversibles en la anatomía, la bioquími$\mathrm{ca}$, y las propiedades biomecánicas de la dentina, que constituye el grueso de la estructura dentaria remanente después de la pérdida pulpar y el tratamiento de endodoncia. ${ }^{1}$

La arquitectura dental es modificada durante el tratamiento endodóntico como resultado de la remoción de caries, el acceso al conducto, y la instrumentación en el canal radicular ${ }^{2}$. La resistencia a la fractura es directamente proporcional al volumen y a la estructura dental remanente; estas características pueden incrementar el riesgo a la fractura. ${ }^{3}$ En 1972, Helfer et al. determinaron el contenido de humedad de los dientes vitales y sin pulpa en perros dividiendo el contenido total de agua de cada diente en agua libre y ligada. En este estudio se demostró que había un 9\% menos de humedad en los tejidos calcificados de los dientes del perro sin pulpa que en los dientes vitales. ${ }^{4}$

Para la rehabilitación de un órgano protésico tratado endodónticamente, la elección de un sistema restaurativo que devuelva a la estructura remanente propiedades de soporte y estabilidad es hoy en día uno de los muchos temas a debatir en la rehabilitación bucal. Los postes intraradiculares o endopostes metálicos han sido la alternativa más utilizada por el clínico durante años. Siendo importante el contemplar el grado de destrucción coronaria y el plan de tratamiento para cada órgano dentario, tomando en cuenta que todo diente endodonciado que soporte una prótesis fija y cumpla la función de pilar protésico, deberá ser reforzado con un endoposte. ${ }^{5}$

El uso de postes metálicos requiere una restauración temporal posterior a la preparación del canal radicular y por lo tanto incrementa el riesgo de reinfección. ${ }^{6-7}$ En contraste, los postes prefabricados pueden ser cementados inmediatamente después de la preparación para el espacio del poste. Desde hace algunos años ha sido cuestión de debate, que los endopostes metálicos pueden llegar a causar fracturas verticales, dando como consecuencia la extracción del órgano dentario y fracaso del tratamiento. ${ }^{8}$ El uso de postes intra-radiculares con un módulo similar de elasticidad a la dentina, como lo son los postes de fibra de vidrio, favorece la distribución del estrés en la estructura dental remanente y a lo largo de la interface de cementación. . $^{9-10}$ Se ha sugerido el uso de estos postes con cementos a base de resina, incrementando la resistencia a la fractura radicular. ${ }^{2-9-11-12}$

Para la prevención de fracturas radiculares, el uso de postes de fibra ha sido recomendado, basado en consideraciones teóricas y en análisis de elementos finitos. Duret et al. ${ }^{13-14}$ postuló que los postes deberían igualar el tejido a restaurar, i.e. dentina, tan cerca como sea posible, especialmente respecto a los módulos de elasticidad. Como resultado, cargas aplicadas a la restauración son uniformemente distribuidas en el soporte dentinario y las concentraciones 
de estrés en la interface de la restauración siendo presumiblemente evitados.

Los postes prefabricados reforzados y cementados con resina son ahora una buena alternativa al tratamiento, al tener propiedades importantes como son la resistencia y flexibilidad similar con las estructuras dentinarias. Aunado a esto, se ha descrito que existe una mejor distribución de estrés como resultado de las fuerzas masticatorias en comparación con los endopostes metálicos. ${ }^{8-15} \mathrm{El}$ objetivo de este trabajo es describir las características físicas de los postes de fibra de vidrio y los postes metálicos, realizar una comparativa y establecer la mejor opción como tratamiento protésico.

| | | | | | | | | | | | | | | | | | | | | | | | | | | | | | | | | | | | | | | | | | | | | | | | | | | | | | | | | | | | | | | | | | | | | | | | | | | | | | | | | | | | | | | | | | | | | | | | | | | | | | | | | | | | |

\section{Material y métodos}

Se realizó una revisión bibliográfica actualizada de la base de datos certificada por la U.S. National Library of Medicine, referentes al tema los cuales describen información acerca de los postes metálicos y de fibra de vidrio para realizar este trabajo comparativo.
La búsqueda fue desarrollada usando una variedad de palabras clave incluyendo glass fiber post, metal alloy, bond strenght, cast post, fracture resistance, luting agent, post and core technique, endodontically treated theet, flexural properties of post materials.

\section{| | | | | | | | | | | | | | | | | | | | | | | | | | | | | | | | | | | | | | | | | | | | | | | | | | | | | | | | | | | | | | | | | | | | | | | | | | | | | | | | | | | | | | | | | | | | | | | | | | | | | | | | | ||}

\section{Resultados y discusión}

Cualquier rehabilitación bucal tiene como objetivo el restaurar y devolver al órgano dentario sus características y funcionalidad, lo más posible a las condiciones naturales y de salud del sistema estomatognático. Los órganos dentarios a los que se les practica una endodoncia quedan debilitados al perder su vitalidad y resistencia intrínseca debido a la deshidratación de la dentina así como la probable pérdida de estructura (caries extensa, traumatismo o preparación protésica) a la que quedan sujetos. ${ }^{5-8}$ El poste es una restauración compuesta de un perno que se localiza en el conducto de una raíz preparada y un muñón localizado en la zona externa que reemplaza la porción coronal que se ha perdido. Se realiza en materiales rígidos que al ser cementados en el conducto radicular y la cámara pulpar brinda una base sólida retenida en el diente. ${ }^{16}$
La restauración utilizando un poste no es la excepción, por lo que se debe de buscar un material que sea lo más parecido al tejido dentinario en aspectos tan importantes como la flexibilidad, dureza y distribución de estrés ante las fuerzas masticatorias para obtener éxito en el tratamiento.

A lo largo de la historia nos hemos encontrado con una gran variedad de formas de endopostes desde madera en los inicios de la odontología moderna, diferentes aleaciones metálicas y ahora se evalúan las propiedades de los postes prefabricados de nueva generación como son fibra de vidrio, fibra de carbono, acero inoxidable reforzado con resina. Lo que buscamos con el endoposte son 2 funciones esenciales: retener la restauración final y la de conservar la estructura dental remanente ya que, cuando se ha perdido el total de la porción coronal del diente, la responsabilidad 
de mantener la integridad de los márgenes está dada por la porción coronal del núcleo ya que las fuerzas oclusales son dirigidas a lo largo de la longitud del poste, lo cual provee alivio del estrés en los márgenes ${ }^{17}$.
La mayoría de los sistemas permiten esto pero investigaciones han demostrado las desventajas y complicaciones clínicas de algunos.

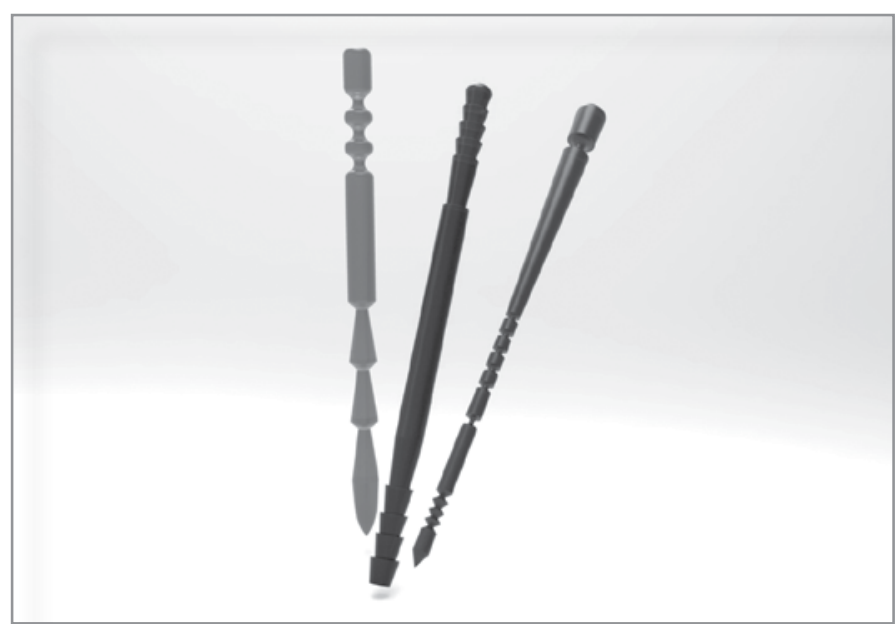

Representación gráfica de diferentes formas de postes de fibra de vidrio.

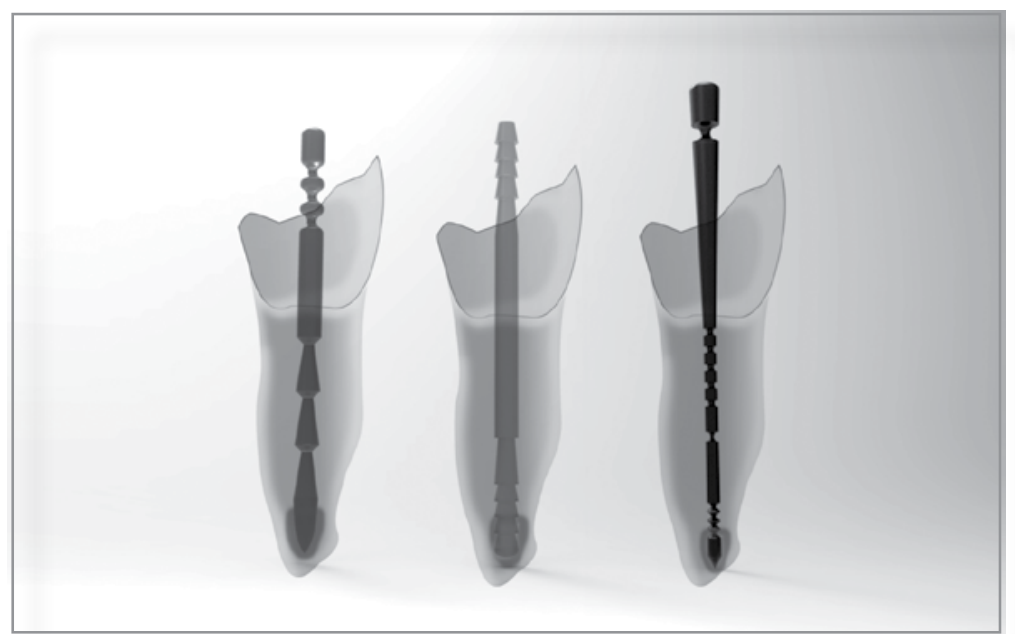

Representación gráfica de diferentes formas de postes de fibra de vidrio dentro del órgano dental.

\section{Módulos elásticos y resistencia a la flexión}

La rigidez de un retenedor intraradicular es la capacidad de soportar cargas sin generar distorsión de los márgenes de la restauración durante la función masticatoria dando como resultado daño en el cemento y caries recurrente. El módulo elástico se refiere a la relación entre el esfuerzo y la deformación o el límite de elasticidad de un material específico, cuando se aplica una fuerza externa que crea una tensión en el interior del material y, así, éste pueda 
regresar a su forma original. El resultado de la medición de estas características nos puede indicar qué sistema proporciona mejores resultados. De la misma manera se ha demostrado que no sólo en la presión única de carga, si no modificándola en ciclos tratando de emular la acción masticatoria de mejor forma, los resultados favorecen a los postes de fibra de vidrio por encima de los de titanio y los de oro. ${ }^{18}$

A continuación (tabla 1) se presentan mediciones de algunos materiales en comparación con la dentina.

\section{Tabla I}

\section{Módulos elásticos y resistencia a la flexión}

\begin{tabular}{|lcc|}
\hline & Módulo Elástico & Resistencia a la flexión \\
\hline Dentina 18 & 17.5Gpa. & $212.9 \mathrm{Mpa}$. \\
\hline Fibra de vidrio 18-19 & $24.4-29.2 \mathrm{Gpa}$. & $879.1 \mathrm{Mpa}$. \\
\hline Metal 18 & $108.6 \mathrm{Gpa}$. & $1545 \mathrm{Mpa}$. \\
\hline Oro 18-19 & 90Gpa. & $356 \mathrm{Mpa}$. \\
\hline Titanio 18 & $112 \mathrm{Gpa}$ & $1478 \mathrm{Mpa}$. \\
\hline
\end{tabular}

\section{Tabla 2}

\section{Número de ciclos de carga aplicados hasta la fractura}

\begin{tabular}{|lllc|}
\hline Grupo & Oro & Titanio & Fibra de Vidrio \\
\hline 1 & 15,478 & 22,257 & 42,362 \\
\hline 2 & 15,640 & 19,143 & 43,982 \\
\hline 3 & 11,800 & 38,142 & 55,218 \\
\hline 4 & 5,672 & 25,020 & 53,818 \\
\hline 5 & 10,894 & 17,360 & 58,102 \\
\hline
\end{tabular}

La tabla 2 nos proporciona el número de ciclos de carga hasta el tiempo de la fractura de los 15 especímenes probados en los 3 grupos. La significancia y las desviaciones estándar de los ciclos a la fractura fueron: Oro 11,897 \pm 4080 ciclos de carga; Titanio $24,384 \pm 8231$ ciclos de carga; y Fibra de vidrio 50,696 \pm 7063 ciclos de carga.

El estudio de ANOVA mostró una diferencia significativa entre los 3 grupos (P.05 $\leq$ ), con el poste de fibra de vidrio exhibiendo el mayor número de ciclos antes del fracaso a la cementación de la corona. ${ }^{19}$

Estos estudios muestran un mejor desempeño en los postes de fibra de vidrio mientras que en metal, los más adecuados para la rehabilitación bucal son los de oro tipo IV. ${ }^{18}$

El tomar en cuenta la estructura dentinaria remanente para la elección del sistema de postes, pensando en un poste de fibra de vidrio sólo si se tiene la estructura de las paredes dentinarias casi intacta, de igual manera queda descartado por otro estudio. ${ }^{20}$

El grupo 1 fue el grupo control, en el grupo 2 fue utilizado un poste prefabricado con estructura remanente de la corona menor de $1 \mathrm{~mm}$, grupo 3 poste prefabricado con estructura remanente de la corona de $1 \mathrm{~mm}$, grupo 4 poste prefabricado con estructura remanente de la corona de $2 \mathrm{~mm}$, grupo 5 poste prefabricado con es- 
tructura remanente de la corona de $3 \mathrm{~mm}$.

La diferencia en los índices de fractura son poco significativos, y la fractura que se puede presentar es siempre en este sistema de postes en situaciones que no implican un fracaso total, siempre con opción a un segundo tratamiento. Caso muy diferente a los postes metálicos, que al centrar el estrés en la parte apical, las fracturas aparecen siempre en esa zona. ${ }^{21-22-23}$

Algunas investigaciones han sugerido a través de diferentes estudios, Daizo $\mathrm{O}$ y cols. ${ }^{20}$ y Yamamoto $M$. y cols. ${ }^{24}$, que mediante resina compuesta como núcleo del muñón y un poste de fibra de vidrio, con una técnica de cementación adecuada, es un tratamiento efectivo para prevenir las concentraciones de estrés ocasionadas por la carga de oclusión en dientes tratados endodónticamente.

\section{Cementación y preparación radicular en postes de fibra de vidrio}

Otro factor a considerar, distinto a la fractura por la rigidez que sucede en los postes de metal, es la retención por el cemento utilizado. El fracaso en la colocación de un poste endodóntico principalmente ocurre en la pérdida de la retención ${ }^{25}$, debido a la calidad de la hibridación del material de cementación, de acuerdo a las desfavorables condiciones de la técnica causadas por un inadecuado acceso y el tipo de poste utilizado. ${ }^{26-27}$

La retención del poste puede ser mejorado por los sistemas de cementación de resina pudiendo ser influenciada por la composición, mecanismo de curado, proceso de polimerización y la técnica de aplicación. Algunas investigaciones han reportado métodos de reforzamiento en paredes dentinarias en el conducto de raíces delgadas con resina compuesta. ${ }^{28-29}$
Sugieren que una adecuada fotopolimerización de resina compuesta en el conducto radicular con postes translúcidos mejoraría la resistencia a la fractura. La dentina y la resina compuesta tienen módulos de resistencia y flexión similares, por lo tanto si la resina compuesta es polimerizada y adherida adecuadamente a la dentina, la estructura del remanente dental será reforzada por la integración de la dentina y resina. ${ }^{30}$ El agente utilizado para cementar es también de vital importancia. Con éste no sólo se evita el desalojo del poste si no que ayuda en gran manera a la resistencia a la fractura de la raíz. ${ }^{31-2}$ Morgano y cols. ${ }^{5}$ analizaron la tasa de fracaso de un poste en el que tan solo el 3.2 fue reportado por desalojo del mismo.

La preparación de un órgano dentario para colocar un poste puede causar que se retire estructura dentinaria de la porción radicular de manera excesiva, evitando así la opción de un retratamiento por lo adelgazado de las paredes. 5, 32

Un incremento en los módulos elásticos del agente cementante dan lugar a resultados sobre la disminución en concentraciones de estrés en el área cervical. Estos resultados son consistentes en previos estudios. $^{33-34}$

Un alto valor de estrés es un fuerte indicador con una mayor posibilidad a la fractura, haciendo importante el reducir estrés en el área cervical. Estudios previos han mostrado que usando agentes cementantes con módulos de elasticidad similares a la dentina, un excesivo nivel de estrés en el área cervical puede ser evitado. ${ }^{33-34}$ Por otro lado Mezzomo et $\mathrm{al}^{35}$, encontró que el tipo de agente cementante usado no es un factor determinante en la resistencia física a la fractura del diente restaurado con postes y coronas de oro fundido. Naumann et $\mathrm{al}^{36}$ y Aggarwal et $\mathrm{al}^{37}$ encontraron que los cementos a base de resina mejoran la resistencia a la fractura de los postes, comparados con cementos como el fosfato de zinc 
o el ionómero de vidrio, pero una técnica adhesiva dificulta limpiar residuo dentinario y remoción de humedad del canal radicular, siendo ésta el principal fracaso de los postes prefabricados.

En el caso de los postes metálicos, al ser realizados se necesita forzosamente que sea 2/3 la longitud total de la raíz mientras que en los postes de fibra de vidrio, no se encontraron diferencias significativas cambiando la longitud de $7 \mathrm{~mm}$ a $13 \mathrm{~mm}$ en O.D. cuya longitud promedio era de 18 $\mathrm{mm}$. Existió incluso un número de casos en los que los postes realizados a menor longitud eran superiores a su contraparte de $13 \mathrm{~mm} \cdot{ }^{38-39}$

\section{Predicción de los factores de comportamiento de la rehabilitación intrarradicular}

Los objetivos de la odontología conservativa son preservar la mayor cantidad de estructura dental posible o la mínima necesaria para la retención del órgano dental en boca. Cuando el diente ha perdido una considerable porción de su estructura, métodos auxiliares son requeridos para asegurar la retención del material de relleno. Los postes intraradiculares son usados para este propósito en los dientes que han sido tratados endodóticamente. ${ }^{40}$

Las ventajas de los postes de fibra de vidrio contra los de acero han sido probadas en numerosos estudios que demuestran el éxito clínico de los sistemas de postes implantados por técnicas adhesivas. Dicho éxito está dado por la excelente retención de las propiedades mecánicas como resultado del bajo módulo de elasticidad, el cual es similar a la dentina: ${ }^{18-42} \mathrm{Gpa} .{ }^{41-42}$

Este comportamiento biomecánico evita la aparición de fracturas radiculares. ${ }^{43-44}$ No obstante, la insuficiente rigidez del poste podría conducir a una excesiva deformación y zonas de concentración de estrés durante la función, ocasionando un riesgo de fracaso marginal. ${ }^{45-46}$

Muchos factores pueden influenciar la supervivencia intrarradicular de la restauración del poste retenido. Primeramente, existen factores dentales, uno de ellos es la medida del efecto férula, el cual como mínimo debe tener 1.5-2 mm de longitud ${ }^{46} \mathrm{y}$ ciertas condiciones como un estatus periodontal sano, relación de dientes adyacentes y antagonistas, parafunciones o hábitos que pueden constituir contradicciones para la restauración del órgano dental. Secundario a esto, existen factores anexos al poste: longitud, conicidad, diámetro y el material del cual está hecho el poste. Hoy en día encontramos una gran variedad de postes disponibles: redondo u ovalados, con diferentes grados de conicidad ${ }^{49}$, mayor o menor translucidez para mejorar la transmisión de luz del fotocurado y el material de los diferentes postes: fibra de vidrio, fibra de carbono, fibra de cuarzo, sílice o híbridos. También, la integridad de superficie es indispensable para los postes de fibra para la adhesión al cemento a base de resina. ${ }^{45}$ Por último, tenemos los factores relacionados al cemento: contracción al curado, dificultades por la luz para fotocurar en las zonas apicales, incompatibilidad química con el sistema adhesivo. ${ }^{47-48}$ Una capa excesiva de cemento alrededor del poste ha sido asociado con una alta frecuencia de postes descementados, debido al incremento de poros y espacios entre el cemento y la dentina radicular, ${ }^{49}$ el grosor y concentración del cemento depende del área y forma radicular, así como del material empleado. 


\section{Conclusiones}

Regularmente las nuevas tendencias en la odontología tardan unos años en consolidarse o quedar descartadas por completo debido al grado de eficiencia y éxito clínico en los tratamientos. Los postes prefabricados (fibra de vidrio, fibra de zirconio, titanio) son relativamente nuevos y están comenzando a ser usados por los clínicos como una nueva alternativa al tratamiento. Por lo cual su etapa de prueba puede estar llegando a su fin.

Hoy en día nos encontramos con una vasta bibliografía y reportes científicos en el que nos muestran las cualidades y grandes ventajas de los postes prefabricados sobre los metálicos. El menor número de casos clínicos, fracturas de raíz o desalojo de la prótesis son algunas que pudimos mencionar en este apartado. El constante avance en la tecnología así como las investigaciones recientes nos colocan en un punto de cambio. Los postes metálicos utilizados en las últimas décadas están por desaparecer. El fin de todo cambio es el de mejorar, utilizando las diferentes opciones posibles para un tratamiento y así obtener una mayor tasa de éxito. Es por esto que se encuentra conveniente analizar toda la información pertinente para poder hacer correcto uso de este nuevo sistema y seguir ofreciendo la mejor opción a los pacientes.

A través de la bibliografía revisada se sugiere una rehabilitación en tratamientos endodónticos que incluya la colocación de un poste de fibra de vidrio aunado a un agente cementante a base de resina y la reconstrucción del núcleo mediante resina compuesta con un módulo de elasticidad similar de los materiales utilizados, logrando minimizar y distribuir la concentración de estrés ocasionadas por fuerzas masticatorias alrededor de la raíz y al término del poste, indicando una menor posibilidad de fracaso.

\section{Bibliografía}

1. Gutmann JL. The dentin-root complex: Anatomic and biologic considerations in restoring endodontically treated teeth. J Prosthet Dent 1992;67:458-67.

2. Meira JB, Espósito CO, Quitero MFZ, Poiate IA Pfeifer CS, Tanaka CB, et al. Elastic modulus of posts and the risk of root fracture. Dent Traumatol. 2009;25:394-8.

3. Egilmez F, Ergun G, Cekic-Nagas I, Vallittu PK, Lassila LV. Influence of cement thickness on the bond strength of tooth-colored posts to root dentin after thermal cycling. Acta Odontol Scand. 2013; 71: 175-82.

4. Helfer AR, Melnick S, Schilder H. Determination of the moisture content of vital and pulpless teeth. Oral Surg Oral Med Oral Pathol 1972;34:661-70.

5. Morgano SM. Restoration of pulpless teeth: Application of traditional principles in present and future contexts. J. Prosthet Dent 1996; 75: 375-380.

6. Demarchi MG, Sato EF. Leakage of interim post and cores used during laboratory fabrication of custom posts. J Endod 2002;28:328-9.

7. wu MK, Pehlivan Y, Kontakiotis EG, Wesselink PR. Microleakage along apical root fillings and cemented posts. J Prosthet Dent 1998;79:264-9

8. Suzuki T, Miura H, Okada D, et al. Investigation of stress distribution in roots restored with different crown materials and luting agents. Dent Mater J. 2008; 27: 229-236.

9. Barjau-Escribano A, Sancho-Bru JL, Forner-Navarro L, Rodríguez-Cervantes PJ, Pérez-Gónzález A, Sanchez-Marín FT. Influence of prefabricated post material on restored teeth: fracture strength and stress distribution. Oper Dent. 2006;31:47-54.

10. Watanabe $M U$, Anchieta RB, Rocha EP, Kina S, Almeida EO, Freitas AC Jr, et al. Influence of crown ferrule heights and dowel material selection on the mechanical behavior of root-filled teeth: a finite element analysis. J Prosthodont. 2012;21:304-11.

11. Dietschi D, Duc O, Krejci I, Sadan A. Biomechanical considerations for the restoration of endodontically treated teeth: a systematic review of the literature, Part II (Evaluation of fatigue behavior, interfaces, and in vivo studies). Quintessence Int. 2008;39:11729.

12. Pegoretti A, Fambri L, Zappini G, Bianchetti M. Finite element analysis of a glass fibre reinforced composite endodontic post. Biomaterials. 2002;23:2667-82.

13. Duret $B$, Reynaud $M$, Duret $F$. Un nouveau concept de reconstitution corono-raduculaire: le Composi- 
post (1). Chir Dent Fr 1990:60:131-41.

14. Duret $B$, Duret $F$, Reynaud $M$. Long-life physical property preservation and postendodontic rehabilitation with the composipost. Compend Contin Educ Dent Suppl 1996:S50-6.

15. Garbin $C A$, Spazzin $A O$, Meira-Júnior $A D$, Loretto SC, Lyra AM, Braz R. Biomechanical behaviour of a fractured maxillary incisor restored with direct composite resin only or with different post systems. International Endodontic Journal 2010; 43: 10981107.

16. Jhonson D. Enfoque moderno en prótesis fija. Buenos Aires: Labor: 2003.

17. Angelo A C, Jon P S. Biomechanics in clinical dentistry. Chicago: Quintessence: 1987.

18. Stewardson DA, Shortall AC, Marquis PM, Lumley PJ. The flexural properties of endodontic post materials. Dent Mater. 2010;26(8):730-6.

19. Goto Y, Nicholls Jl, Phillips KM, Junge T. Fatigue resistance of endodontically treated teeth restored with three dowel-and-core systems. The Editorial Council of The Journal of ProstheticDentistry 2005;93(1):4550

20. Daizo O, Hiroyuki M, Chikako S, Wataru K, Chiharu $S$, Masahiro Y. Stress Distribution in roots restored with different types of post systems with composite resin. Dental Materials Journal 2008;27(4): 605611.

21. Fraga RC, Chaves BT, Mello GS, Siqueira JF. . J Oral Rehabil 1998; 25: 809-813.

22. Akkayan B, Gülmez T. Resistance to fracture of endodontically treated teeth restored with different post systems. J Prosthet Dent 2002; 87: 431-437.

23. Tezvergil A, Lassila LV, Vallittu PK. Strength of Adhesive-bonded Fiber-reinforced composites to enamel and dentin substrates. J Adhes Dent 2003;5(4):301311.

24. Yamamoto $M$, Miura $H$, Okada $D$, Komada $W$, Masuoka D. Photoelastic stress analysis of different post and core restoration methods. Dent Mater J 2009;28(2):204-211

25. B. Bergman, P. Lundquist, U. Sjogren, and G. Sundquist, J. Prosthet. Dent. 61, 10 (1989).

26. A. H Hatzikyriakos, G. I. Reisis, and N. Tsingo, J. Prosthet. Dent. 67, 454 (1992).

27. A. Mallmann, L. B. Jacques, L. F. Valandro, P. Mathias, and A. Muench, per. Dent. 30, 500 (2005).

28. Saupe W, Gluskin A, Radke R. Acomparative study of fracture resistance between morphologic dowel and cores and a resin-reinforced dowel system in the intraradicular restoration of structurally compromised roots. Quintessence Int 1996; 27: 483-491.

29. Moosavi H, Maleknejad F, Kimyai S. Fracture resistance of endodontically-treated teeth restored using three root-reinforcement methods. J Contemp Dent Pract 2008; 9: 30-37.

30. Fukui Y, Komada W, Yoshida K, Otake S, Okada D, Miura $\mathrm{H}$. Effect of reinforcement with resin composite on fracture strength of structurally compromised roots. Dental Materials Journal 2009; 28(5): 602609.

31. Zogheib LV, Pereira JR, Valle AL, Oliveira JA, Pegoraro LF. Fracture resistance of weakened roots restored with composite resin and glass fiber post. Braz Dent J. 2008;19(4):329-33.

32. Ferrari M, Vichi A, Mannocci F, Mason PN. Retrospective study of the clinical performance of fiber posts. Am J Dent. 2000;13 (Spec No):9B-13B.

33. Suzuki C, Miura H, Okada D, Komada W. Investiga- tion of stress distribution in roots restored with different crown materials and luting agents. Dent Mater J 2008;27:229-36

34. Suzuki S, Nagai E, Taira $Y$, Minesaki Y.In vitro wear of indirect composite restorates. J Prosthet Dent 2002;88:431-6.

35. Mezzomo E, Massa F, Suzuki RM. Fracture resistance of teeth restored with 2 different post-and-core designs with 2 different luting cements: an in vitro study. Part II. Quintes-sence Int 2006;37:477-84.

36. Naumann $M$, Sterzenbach $G$, Rosentritt $M$, Beuer $F$, Frankenberger $R$. Is adhesive cementation of endodontic posts necessary? J Endod 2008;34:100610.

37. Aggarwal R, Gupta S, Tandan A, Gupta NK, Dwivedi $R$, Aggarwal R. Comparative evaluation of fracture resistance of various post systems using different luting agents under tangential loading. J Oral Biol Craniofac Res 2013;3:63-7.

38. Schiavetti R, García-Godoy F, Toledano M, Mazzitelli C, Barlattani A, Ferrari M, Osorio R. Comparison of fracture resistance of bonded glass fiber posts at different lengths. Am J Dent. 2010;23(4):227-30.

39. Hsu ML, Chen CS, Chen BJ, Huang HH, Chang CL.Effects of post materials and length on the stress distribution of endodontically treated maxillary central incisors: a 3D finite element analysis.J Oral Rehabil. 2009;36(11):821-30.

40. Rodriguez-Cervantes PJ, Sancho-Bru JL, BarjauEscribano A, Forner-Navarro L, Pérez AP, SánchezMarín FT, Influence of prefabricated post dimensions on restored maxillary central incisors. J Oral Rehabil. 2007;34:141-52.

41. Lasilla LV, Tanner J, Le Bell AM, Narva K, Vallitu PK, Flexural properties of fiber reinforced root canals posts. Dent Mater. 2004;20:29-36.

42. Grande N, Butti A, Plotino G, Somma F. Adapting fiber-reinforced composite root canal post for use in noncircular-shaped canals. Pract Proced Aesthet Dent. 2006;18:593-9.

43. $D^{\prime}$ Arcangelo $C$, Cinelli $M$, De Angelis $F, D^{\prime}$ Amario $M$. The effect of resin cement film thickness on the pull-out strength of a fiber-reinforced post system. J Prosthet Dent. 2007; 98:193-8.

44. Barjau- Escribano A, Sancho- Bru JL, Forner- Navarro L, Rodriguez- Cervantes PJ, Pérez- González A, Sánches Marín FT. influence of prefabricated post material on restored teeth: fracture strength and stress distribution. Oper Dent. 2006; 31:47-54.

45. Plotino G, Grande NM, Pameijer $\mathrm{CH}$, Somma F. Influence of surface remodeling using burs on the macro and micro surface morphology of anatomically formed fiber posts. Int Endod J. 2008;41:345-55.

46. Al-Omiri MK, Mahmoud AA, Rayyan MR, AbuHammad O. Fracture resistance of teeth restored with post-retained restorations: an overview. J. Endod. 2010;36:1439-49.

47. Bonfante EA, Pegoraro LF, de Góes MF, Carvalho RM. SEM observation of the bond integrity of fiberreinforced composite posts cemented into root canals. Dent Mat. 2008;24:483-91.

48. Perez BE, Barbosa SH, Melo RM, Zamboni SC, Ozcan $M$, Valandro LF, et al. Does the thickness of the resin cement affect the bond strength of a fiber post to the root dentin? Int J Prosthodont. 2006; 19:606-9.

49. Grandini S, Goracci C, Monticelli F, Borracchini A, Ferrari $M$. SEM evaluation of the cement layer thickness after luting two different posts. J Adhes Dent. 2005; 7:235-40. 